\title{
Imiter la réponse immunitaire \\ humorale polyclonale
}

> Les anticorps monoclonaux ont révolutionné le traitement de nombreuses maladies mais leur efficacité clinique reste limitée dans certains cas. Des associations d'anticorps se liant à une même cible (homo-combinaisons) ou à plusieurs cibles différentes (hétéro-combinaisons), mimant ainsi une réponse immunitaire humorale polyclonale, ont conduit à une amélioration thérapeutique dans des essais précliniques et cliniques, essentiellement en cancérologie et en infectiologie. Ces combinaisons augmentent l'efficacité des réponses biologiques et court-circuitent les mécanismes de résistances observés lors d'une monothérapie par anticorps. Le procédé de formulation et d'administration des combinaisons d'anticorps le plus fréquent est une formulation séparée, avec injection séquentielle de chaque anticorps «principe actif». Alternativement, se développent des formulations combinées, où les anticorps produits séparément sont mélangés avant administration, ou produits simultanément par une lignée cellulaire unique ou un mélange de lignées cellulaires correspondant à une master-bank cellulaire polyclonale. La réglementation, la toxicité et la séquence d'injection des mélanges oligoclonaux restent des points à éclaircir et à optimiser pour un meilleur effet thérapeutique. <

Au XIX siècle, les travaux pionniers de Shibasaburo Kitasato et $\varepsilon$ mil von Behring en Allemagne, et d'Emile Roux en France, ouvrirent la voie à la sérothérapie, qui consiste en l'utilisation à but thérapeutique de sérums d'animaux ou d'hommes préalablement immunisés, contenant des anticorps spécifiques de pathogènes comme principe actif. César Milstein et Georges Köhler ont révolutionné ce concept en inventant la technique d'hybridation lymphocytaire

\section{De l'association de deux anticorps monoclonaux aux productions oligoclonales} Christel Larbouret ${ }^{1}$, Marie-Alix Poul ${ }^{1}$,
Thierry Chardès ${ }^{1,2}$

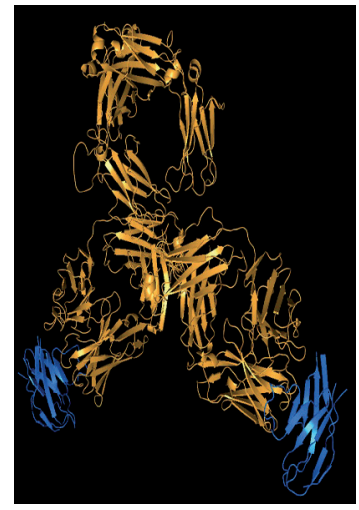

${ }^{1}$ Institut de Recherche en Cancérologie de Montpellier (IRCM), Inserm U1194, Université de Montpellier, Institut régional du Cancer de Montpellier (ICM), 34298 Montpellier, France. ${ }^{2}$ Centre National de la Recherche Scientifique (CNRS), Paris, France.

thierry.chardes@inserm.fr

et ont permis le développement d'une nouvelle classe pharmacologique de biomédicaments, les anticorps monoclonaux (AcM) qui font l'objet de ce numéro de médecine/sciences. Toutefois, les réponses partielles et éphémères, souvent associées à des phénomènes de résistance (bien étudiés en recherche fondamentale), limitent l'efficacité clinique des AcM. Pour pallier ces obstacles, des associations d’AcM, le plus souvent évalués séparément ou oligoclonaux, cocktail d'anticorps considéré comme un biomédicament unique, sont en cours de développement. En effet, le système immunitaire a naturellement évolué pour développer une réponse humorale polyclonale, optimisant ainsi sa capacité à combattre les maladies, plutôt qu'une stratégie monoclonale telle que proposée par les biomédicaments anticorps actuellement approuvés. L'autorisation de mise sur le marché (AMM) de deux combinaisons d'anticorps thérapeutiques a récemment validé ce concept de «mime » de la réponse immunitaire humorale polyclonale en oncologie. Les améliorations techniques dans la capacité à produire des mélanges oligoclonaux reproductibles d'anticorps, chaque composant étant sélectionné sur des critères précis, tels que la spécificité épitopique, l'affinité ou son activité biologique intrinsèque, permettent maintenant d'imiter la réponse immunitaire humorale polyclonale, ouvrant ainsi la voie à la sérothérapie du $x x I^{e}$ siècle. 


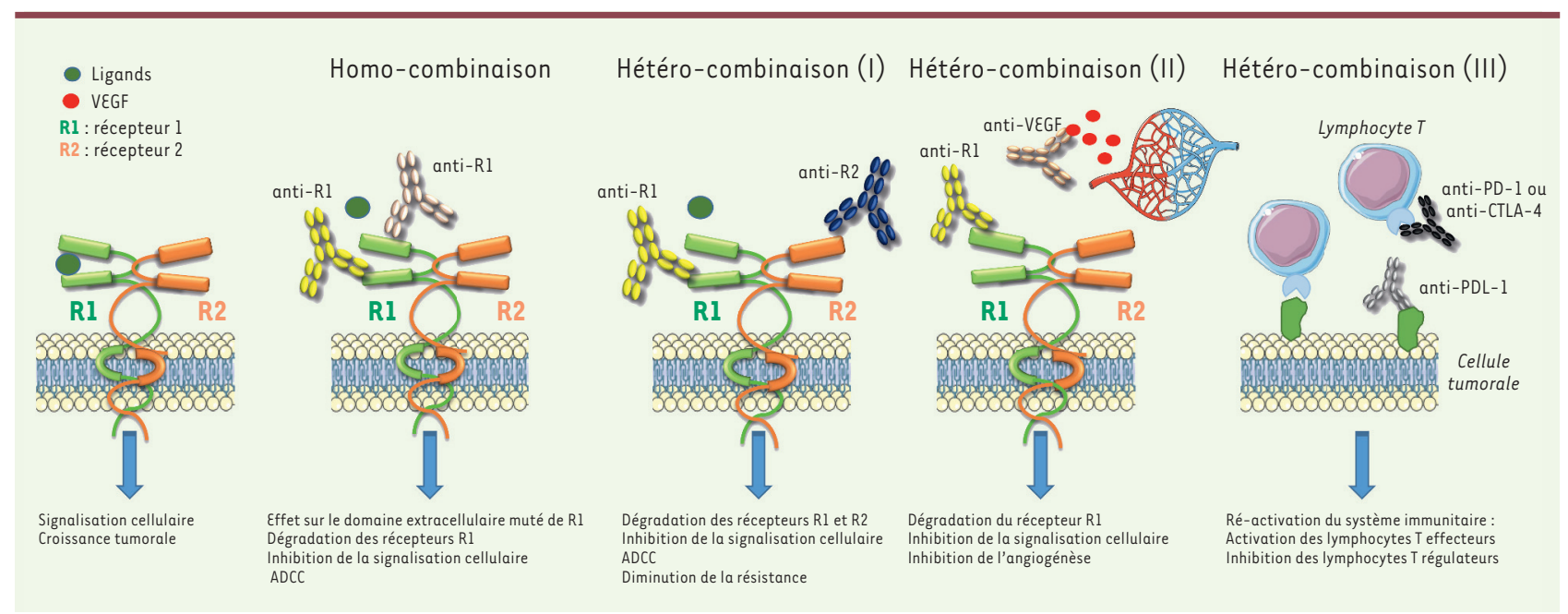

Figure 1. Les homo- ou hétero-combinaisons d'anticorps monoclonaux (adapté d'après [55]).

\section{Les prémices d'un mélange oligoclonal thérapeutique maitrisé : homo-combinaison et hétéro-combinaison d'anticorps en préclinique}

\section{Co-ciblage anti-tumoral}

Autour des années 2000, le concept d'homo-combinaison d'anticorps, engageant des épitopes distincts sur un même récepteur, a été proposé par plusieurs équipes, dont celle de Yosef Yarden (institut Weizmann, Israël). Ainsi, des homo-combinaisons d'anticorps anti-EGFR (epidermal growth factor receptor) [1], anti-HER2 (human epidermal growth factor receptor-2) [2] ou anti-cMET (ou hepatocyte growth factor [HGF] receptor) [3] induisent une activité anti-tumorale synergique due à l'accélération de la dégradation des récepteurs ciblés et à une cytotoxicité à médiation cellulaire dépendant des anticorps (ADCC) renforcée (Figure 1). Ces combinaisons permettent de contrer la résistance au traitement provoquée par une monothérapie anti-£GFR dans le cancer colorectal [4], ou anti-cMET dans le cancer gastrique [5], et de maintenir une activité anti-tumorale malgré la présence de mutations des domaines extracellulaires de ces récepteurs [6]. Dès 2007, notre équipe à l'institut de recherche en cancérologie de Montpellier [7], démontrait que I'hétéro-combinaison d'anticorps anti-EGFR/ anti-HER2 engageant des épitopes distincts sur ces deux récepteurs collaborant fonctionnellement, induisait une synergie anti-tumorale en favorisant l'ADCC, la diminution de l'expression de ces récepteurs et la formation des homodimères, mais aussi en inhibant les voies de signalisation intracellulaires [8]. Ces travaux précliniques, confirmés par d'autres groupes de recherche [9], ont permis la mise en place de l'essai clinique de phase I THERAPY dans le cancer du pancréas métastatique et progressant sous gemcitabine. L'association cétuximab/trastuzumab (qui ciblent respectivement l'EGFR et HER2) a induit une stabilisation de la maladie chez $27 \%$ des patients, sans réponse objective, mais avec une corrélation positive entre une toxicité cutanée et la survie sans progression des patients [10]. L'essai n'a pu être poursuivi à cause de cette toxicité, soulignant ainsi la nécessité de repenser la dose active dans le cas de combinaison d'anticorps. Ce concept d'hétéro-combinaison d'anticorps a pour but d'éviter les phénomènes de signalisation compensatoire liés au ciblage par les anticorps d'un récepteur unique. Il a été étendu au double ciblage EGFR/HER3 dans les tumeurs résistantes au cétuximab [4]. Ces hétéro-combinaisons d'anticorps peuvent également associer le ciblage d'un ligand, tel que le VEGF (vascular endothelial growth factor) ou l'HGF, pour agir sur le microenvironnement, et le ciblage d'un récepteur spécifique de la tumeur [11]. Enfin, des cocktails oligoclonaux de trois [12] ou six [13] anticorps ciblant respectivement l'EGFR, HER2 et HER3 ont démontré un effet anti-tumoral accru dans des modèles expérimentaux, avec un blocage des voies de signalisation sousjacentes ERK (extracellular signal-regulated kinases) et AKT (protéine kinase $B$ ), et une dégradation accélérée des récepteurs. L'homo- ou l'hétéro-combinaison d'anticorps en hémato-oncologie a également été proposée à l'aide d'anticorps ciblant les molécules CD20, CD22 ou CD52 exprimées par les lymphocytes B (et T en ce qui concerne CD52) [14].

\section{Co-ciblage anti-microbien}

Reproduire la réponse immunitaire humorale par une combinaison d'anticorps thérapeutiques trouve aussi tout son sens dans le cadre d'une thérapie anti-infectieuse. En effet, les virus et les bactéries sont complexes (souches, facteurs de virulence, variabilité, etc.) et adoptent de nombreuses stratégies d'échappement et ont des mécanismes d'action variés. Dès 1982, Volk et al. montrent que la combinaison de deux anticorps ciblant le virus de la stomatite vésiculaire améliore la neutralisation virale [15]. Depuis lors, des anticorps oligoclo- 
naux ciblant le VIH (virus de l'immunodéficience humaine) [16], le virus Ebola [17], le virus de l'hépatite B [18], le virus de la vaccine [19], le MERS-coronavirus (responsable du syndrome respiratoire du MoyenOrient) [20], le virus de la rage [21], ou des toxines des bactéries Clostridium difficile [22], de C. botulinum [23] ou de Bordetella pertussis [24], entre autres, ont démontré une synergie dans leur capacité de neutralisation. Toutes ces démonstrations précliniques ont largement souligné l'intérêt de combiner des anticorps thérapeutiques pour imiter la réponse immunitaire humorale polyclonale.

\section{Co-ciblage des molécules de contrôle immunitaire : réveiller le système immunitaire}

L'importance des points de contrôle immunitaire, telles que CTLA-4 (cytotoxic T lymphocyte-associated protein 4) et PD-1 (programmed cell death 1 ), pour moduler la réponse T anti-tumorale a été soulignée récemment par l'attribution du prix Nobel de physiologie ou médecine 2018 à James Allison et Tasuku Honjo. La compréhension de leurs rôles dans la régulation de l'activation des lymphocytes et dans l'échappement immunitaire des tumeurs a conduit au développement d'associations d'anticorps ciblant les molécules de cette famille fonctionnelle, qui sont classées en molécules co-inhibitrices (que l'on cherche à bloquer) ou molécules co-activatrices (que l'on cherche à stimuler). L'hétéro-combinaison d'anticorps anti-CTLA-4/anti-PD-1 bloquants a conduit dès 2010 à valider une synergie thérapeutique anti-tumorale dans des modèles murins de cancer colorectal et de mélanome, marquée par une infiltration accrue de lymphocytes $T$ cytotoxiques et une inhibition de lymphocytes T régulateurs et de cellules myéloïdes suppressives [25]. D'autres hétéro-combinaisons d'anticorps anti-points de contrôle immunitaire PD-1/4-1-BB (CD137 ou tumor necrosis factor receptor superfamily member 9) ou PD-1/LAG3 (lymphocyte-activation gene 3) [26], TIM3 (T-cell immunoglobulin and mucin-domain containing-3) [27], entre autres, ont été proposées pour moduler les réponses immunitaires dans les cancers. Le lien, récemment démontré, entre l'expression ou la surexpression de PD-Ll (le ligand de PD-1) chez certains patients et l'efficacité d'anticorps anti-HER2/Neu [28] suggère qu'il serait intéressant de combiner des anticorps ciblant des membres de la famille HER avec des anticorps ciblant les points de contrôle immunitaires dans certains cancers.

\section{La preuve de concept clinique : trastuzumab/pertuzumab dans le cancer du sein et nivolumab/ipilimumab dans le mélanome}

La première association d'anticorps approuvée en 2012 a été l'homocombinaison anti-HER2 trastuzumab/pertuzumab associée au docétaxel $^{1}$ dans le cancer du sein métastatique HER2-amplifié. L'essai de phase III CLEOPATRA, portant sur 808 patientes, a montré une survie moyenne sans progression (PFS) de 18,7 mois dans le groupe traité par la combinaison anticorps + docétaxel contre 12,4 mois dans le groupe traité par le trastuzumab seul + docétaxel [29], avec une toxicité cardiaque comparable dans les deux bras de traitement. Cette combi-

${ }^{1}$ Alcaloïde qui se lie à la tubuline, favorisant sa polymérisation en microtubules. naison associée au docétaxel a ensuite été approuvée en traitement néo-adjuvant chez des patientes nouvellement diagnostiquées ( $\varepsilon$ ssais APHINITY et NEOSHERE [30]). Toutefois, l'essai clinique NEOSPHERE a révélé une plus forte toxicité dans le bras traité par la combinaison trastuzumab/pertuzumab. Tous les essais cliniques de phase III associant des anticorps n'ont cependant pas conduit à des résultats positifs. Ainsi, dans le cancer gastrique HER2-positif (étude JACOB), I'association pertuzumab et trastuzumab avec le cisplatine ou le 5-fluoro-uracile n'a pas amélioré la survie des patients [31]. L'étude CAIR02 [32], associant I'hétéro-combinaison bévacizumab/cétuximab avec l'oxaliplatine et la capécitabine, l'étude PACCE [33] impliquant bévacizumab/panitumumab associé à l'oxaliplatine et l'irinotécan dans le cancer colorectal métastatique, et l'étude AVEREL [34], associant trastuzumab et bévacizumab dans le cancer du sein HER2-amplifié, se sont aussi révélées infructueuses. Le co-ciblage du VEGF avec les récepteurs $\varepsilon G F R$ ou HER2 ne semble donc pas pertinent en termes de synergie ou d'additivité, peut-être à cause d'interactions négatives entre voies de signalisation, ou d'interactions pharmacodynamiques (défaut de vascularisation tumorale, inhibition de l'expression d'un des deux récepteurs) [35].

Seuls 20-30\% des patients atteints de mélanome métastatique répondent à la monothérapie avec les anticorps anti-CTLA-4 ou anti-PD-1 bloquant les points de contrôle immunitaire. Dès 2013, un essai clinique de phase I associant I'anti-CTLA-4 ipilimumab avec l'antiPDl nivolumab démontrait une régression tumorale chez $50 \%$ des patients traités [36]. Dans l'essai clinique de phase III Checkmate 067, portant sur 945 patients atteints de mélanome métastatique, la survie sans progression a été supérieure dans le bras traité par l'hétéro-combinaison ipilimumab/nivolumab (11,5 mois), comparativement aux bras traités par le nivolumab (6,9 mois), ou par l'ipilimumab (2,9 mois) [37]. Toutefois, ce bénéfice en ce qui concerne la survie est associé à une toxicité accrue dans le bras traité par l'hétérocombinaison nivolumab/ipilimumab ( $55 \%$ des patients ont des effets secondaires de grade 3 et 4$)$, comparé aux bras monothérapie ( $16 \%$ pour le nivolumab et $27 \%$ pour l'ipilimumab) [38]. Cet essai a conduit la food and drug administration (FDA) américaine à approuver cette combinaison d'anticorps anti-points de contrôle immunitaire dans le mélanome métastatique. Depuis, des essais cliniques de phase III, associant anti-CTLA-4 et anti-PD-1 ou anti-PD-Ll, ont donné des résultats positifs dans le cancer du poumon et le carcinome rénal. Enfin, la combinaison de l'anticorps anti-PD-Ll atézolizumab avec l'anticorps anti-VEGF bévacizumab, 
associée à la chimiothérapie dans le cancer du poumon, a récemment montré un bénéfice en terme de survie sans progression par rapport au bras sans atézolizumab ( 8,3 mois vs 6,8 mois) [39].

Environ 300 essais cliniques ${ }^{2}$ utilisant une combinaison d'anticorps, associée ou non avec une chimiothérapie, sont actuellement en cours dans de nombreuses indications [40-42]. En cancérologie, l'essentiel des études, complètes ou en cours de recrutement, implique l'association ipilimumab/nivolumab (environ 130 essais, dont une cinquantaine dans les indications mélanome et cancer du poumon [42]) et l'association trastuzumab/pertuzumab (environ 40 études [40]). D'autres essais impliquant des homo- ou hétéro-combinaisons sont répertoriés:

- Anti-HER2 trastuzumab/anti-PD-Ll atézolizumab (3 essais),

- Anti-HER2 pertuzumab/anti-PD-Ll atézolizumab (3 essais),

- Anti-EGFR cétuximab/anti-PD-Ll avélumab (4 essais),

- Anti-PD-Ll durvalumab/anti-CTLA-4 trémélimumab (35 essais),

- Anti-VEGF bévacizumab/anti-PD-Ll atézolizumab (16 essais),

- Anti-VEGF bévacizumab/anti-PDl pembrolizumab (6 essais),

- Anti-VEGF bévacizumab/anti-HER2 trastuzumab (4 essais),

- Anti-VEGF bévacizumab/anti-EGFR cétuximab (12 essais),

- ADC trastuzumab-Emtansine/pertuzumab ou trastuzumab ou pembrolizumab ou atézolizumab ou anti-4-BBl (11 essais).

La plupart de ces combinaisons d'anticorps sont administrées séquentiellement, à partir d'entités biologiques anticorps développées individuellement comme biomédicament, et ayant obtenu initialement une AMM en tant qu'anticorps monoclonal « principe actif unique ». Le choix de la séquence de traitement reste encore à optimiser, notamment si des combinaisons d'anticorps anti-tumeur avec des anticorps anti-point de contrôle immunitaire sont proposées. En infectiologie, une vingtaine d'essais cliniques sont recensés (Tableau I). Dans quelques cas, de nouvelles stratégies industrielles de production/ formulation, développées par certaines sociétés pharmaceutiques, ont permis de rationaliser et d'optimiser la production de combinaison d'anticorps (Figure 2).

\section{La validation industrielle des combinaisons d'anticorps: formulation 《unique » ou «séparée », production en « cellule unique » ou « par un mélange de plusieurs cellules »?}

La production d'anticorps pour développer des associations thérapeutiques peut se réaliser selon 4 stratégies principales (Figure 2), qui ont conduit au développement clinique de mélanges oligoclonaux d'anticorps (Tableau / et II):

- Stratégie «formulation séparée »: les principes actifs « anticorps» sont produits séparément à partir de chaque lignée cellulaire (surtout des cellules $\mathrm{CHO}$ ) ; la formulation pharmaceutique est réalisée individuellement et les biomédicaments anticorps sont injectés séquentiellement lors du traitement. Cette stratégie pharmaceutique est la plus classique lorsqu'on utilise des biomédicaments "anticorps monoclonal » déjà approuvés. Elle est à la base de l'approbation des

\footnotetext{
${ }^{2}$ ClinicalTrials.gov
}

combinaisons trastuzumab/pertuzumab et nivolumab/ ipilimumab par les autorités règlementaires et est utilisée dans la plupart des essais cliniques en cours.

- Stratégie «formulation unique » : les principes actifs «anticorps » sont produits séparément à partir de chaque lignée cellulaire (généralement la lignée $\mathrm{CHO}$ ) et l'étape de formulation pharmaceutique mélange les principes actifs pour conduire au biomédicament « combinaison ». Cette stratégie a été développée pour produire la combinaison Sym004 de 2 anticorps antiEGFR, le futuximab et le modotuximab avec une stœchiométrie 1/1 [43], cette combinaison étant actuellement en essai clinique de phase II dans le cancer colorectal métastatique et le glioblastome. Une autre combinaison de trois anticorps anti-neurotoxine de $C$. botulinum (Xoma $3 A B$ ) a été développée jusqu'en phase I [44]. Cette stratégie a aussi été utilisée pour produire I'anticorps MM-15] [6,45], mélange stœchiométrique 2/2/l de trois anticorps anti-EGFR, testé jusqu'en phase I, en association avec de la chimiothérapie ou avec l'anticorps anti-HER3 MM-121, dans le cancer colorectal et le cancer du poumon.

- Stratégie «production en cellule unique » : Les principes actifs «anticorps» sont produits ensemble par les cellules d'une seule lignée cellulaire. Une formulation pharmaceutique unique conduit au biomédicament « combinaison ». Ce procédé Oligoclonics ${ }^{\circledR}$ [46], utilise la lignée cellulaire PER.C6 transfectée avec une construction génétique codant une seule chaîne légère kappa, et deux constructions génétiques codant chacune une chaîne lourde $\mathrm{H}$ de spécificité différente (technologie « common light chain »). Des mélanges d'anticorps mono-spécifiques et bi-spécifiques ont été développés en utilisant cette approche. À noter que cette technologie a été ensuite associée avec l'ingénierie du domaine $\mathrm{CH} 3$ pour forcer la production préférentielle d'anticorps bi-spécifiques, dont certains sont en cours de développement clinique, tels que les anticorps ciblant HER2/HER3 [47] dans le cancer du sein et le cancer gastrique, EGFR/Lgr5 (leucine-rich repeat containing $G$ protein-coupled receptor 5 ) dans les tumeurs solides, et CD3/CLEC12A (C-type lectin domain family 12 member $A$ ) dans la leucémie aiguë myéloïde. - Stratégie "production par un mélange de plusieurs cellules »: les lignées cellulaires produisant les principes actifs « anticorps » sont initialement mélangées pour produire une «master-cell bank » cellulaire polyclonale. Une formulation unique conduit au biomédicament « combinaison ». Le procédé permet d'intégrer d'une manière site-spécifique chaque construction anticorps sur le même locus chromosomique dans chaque lignée cellulaire (Flp-In, CHO, CHO-DG44) [48], 


\begin{tabular}{|c|c|c|c|c|c|}
\hline Pathogène & Anticorps & Formulation & Cibles & $\begin{array}{l}\text { Phase } \\
\text { clinique }\end{array}$ & Date \\
\hline \multirow[t]{6}{*}{$\begin{array}{l}\text { Virus de l'immunodéfi- } \\
\text { cience humaine }\end{array}$} & $3 B N C 117+10-1074$ & Séparée & $\begin{array}{l}\text { CD4bs-gpl20 + V3 } \\
\text { glycan site Env }\end{array}$ & Phase I & 2018 \\
\hline & VRC01LS + 10-1074 & Séparée & $\begin{array}{l}\text { CD4bs-gpl20+V3 } \\
\text { glycan site Env }\end{array}$ & Phase I/II & 2018 \\
\hline & 10E8VLS + VRC07-523LS & Séparée & gp4l + CD4bs-gpl20 & Phase I & 2018 \\
\hline & VRC01LS + VRC07-523LS & Séparée & $\begin{array}{l}\text { CD4bs-gp } 120+\text { CD4bs- } \\
\text { gpl20 }\end{array}$ & Phase I & 2018 \\
\hline & PGDM1400 + PGT121 & Séparée & $\begin{array}{l}\text { V2-loop Env + V3 } \\
\text { glycan site Env }\end{array}$ & Phase I & 2017 \\
\hline & $2 \mathrm{Gl}+4 \varepsilon 10+2 \mathrm{~F} 5$ & Unique & Env & Phase I/II & 2007 \\
\hline \multirow[t]{2}{*}{ Virus de la grippe } & CT-P27 & Unique & $\begin{array}{l}\text { Hémaglutinines } \\
\text { groupe } 1 \text { et } 2\end{array}$ & Phase IIb & 2018 \\
\hline & CR8020 + CR6261 & Séparée & Hémaglutinines & Phase IIa & 2014 \\
\hline \multirow[t]{4}{*}{ Clostridium difficile } & MK-6072 + MK-3415A & Séparée & Toxines $A$ et $B$ & Phase III & 2018 \\
\hline & MK-3415 + MK-6072 + MK-3415A & Séparée & Toxines $A$ et $B$ & Phase III & 2018 \\
\hline & MK-6072 + MK-3415A & Séparée & Toxines $A$ et $B$ & Phase III & 2018 \\
\hline & GS-CDAl + MDX-1388 & Séparée & Toxines $A$ et $B$ & Phase II & 2010 \\
\hline Shigella & caStxl + CaStx2 & Unique & Toxines Shiga 1 et 2 & Phase II & 2013 \\
\hline \multirow[t]{2}{*}{ Virus de la rage } & SYN023 (CTB011 + CTB012) & Unique & Rabies G protein & Phase I & 2018 \\
\hline & CL184 & Unique & Rabies $\mathrm{G}$ protein & Phase II & 2013 \\
\hline Virus respiratoire syncitial & Motavizumab (MEDI-524) + Palivizumab & Séparée & RSV & Phase II & 2012 \\
\hline \multirow[t]{4}{*}{ Clostridium botulinum } & NTM-1633 (Xع02 + Xع06 + Xع17) & Unique & $\begin{array}{l}\text { Neurotoxines } \varepsilon 1, \varepsilon 3 \\
\text { et } \varepsilon 4\end{array}$ & Phase I & 2018 \\
\hline & NTM-1634 (XC41+XC42+XC44+XC84) & Unique & Neurotoxines $C$ et $D$ & Phase I & 2018 \\
\hline & NTM-1632 (3 Abs) & Unique & Neurotoxine B & Phase I & 2017 \\
\hline & Xoma 3AB & Unique & Neurotoxine A & Phase I & 2014 \\
\hline \multirow[t]{3}{*}{ Virus Ebola } & GamEMab & Unique & Ebola & Phase I & 2018 \\
\hline & Zmapp $(Z m a b+c 2 G 4+c 4 G 7)$ & Unique & Protéine GP & Phase I & 2018 \\
\hline & REGN3470+3471+3479 & Unique & protéine GP & Phase I & 2018 \\
\hline MERS coronavirus & REGN3048 + REGN3051 & Séparée & Protéine Spike & Phase I & 2018 \\
\hline Staphylococcus aureus & ASN $100(A S N-1+A S N-2)$ & Unique & $\begin{array}{l}\text { a-hémolysine } \\
\text { et leukotoxines }\end{array}$ & Phase II & 2018 \\
\hline
\end{tabular}

Tableau I. Principaux essais cliniques utilisant une combinaison d'anticorps en thérapie anti-infectieuse. gp 120 : glycoprotéine d'enveloppe (Env) ; V3 glycan site Env : site de glycosylation de la région V3 de gpl20.

dans le but d'uniformiser le niveau d'expression de chaque anticorps après mélange des lignées cellulaires transformées. Ainsi, un mélange maîtrisé de 25 anticorps anti-RhD (Rhésus D) (rozrolimupab ou
Sym001 [49]) a été produit et testé en phase II dans le purpura thrombocytopénique [50], un mélange de deux anticorps anti-cMET avec une stœchiométrie 1/1 


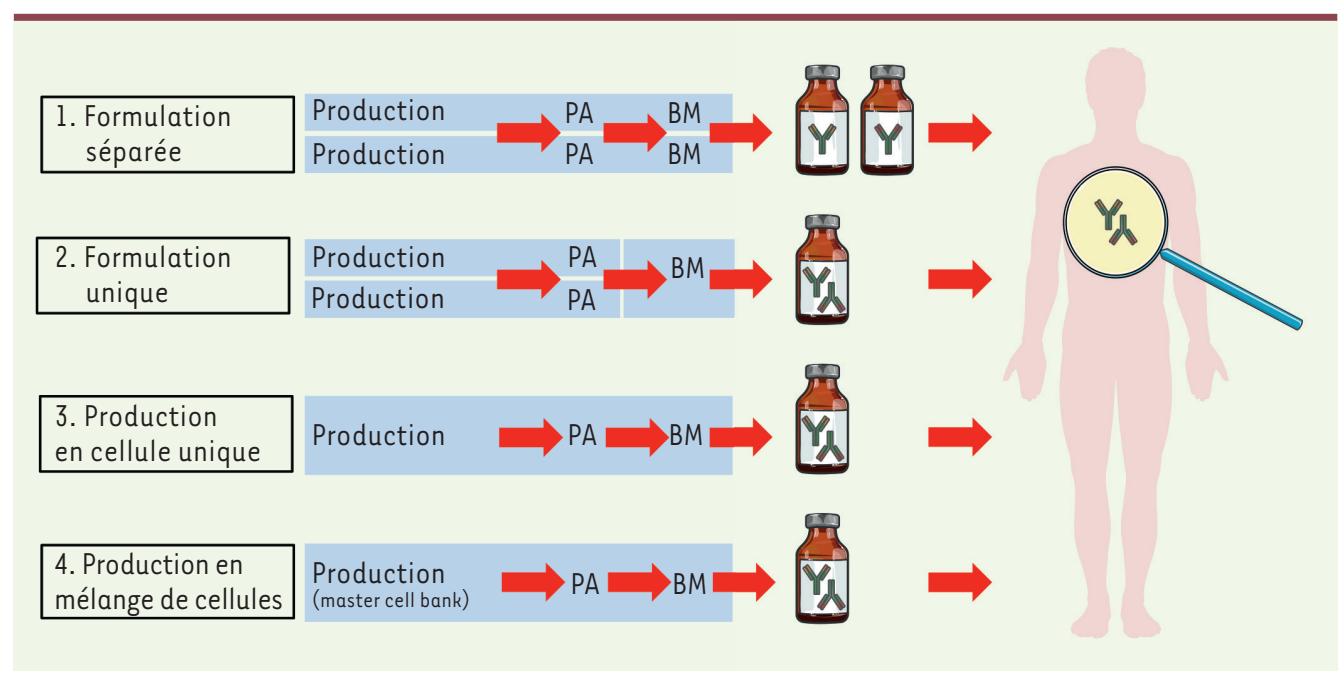

Figure 2. Différentes stratégies pour produire un biomédicament ( $B M$ ) représentant un mélange d'anticorps thérapeutiques, à partir de deux principes actifs (PA) anticorps. La stratégie «Production en cellule unique » permet de produire un mélange d'anticorps mono- ou bi-spécifique (adapté d'après [52]).

(Sym015 [3,5]) en phase II dans les tumeurs MET-amplifiées, et un mélange oligoclonal de deux anticorps anti-EGFR, deux anticorps antiHER2/Neu et deux anticorps anti-HER3 (Sym013 $[4,13]$ ) en phase I dans les cancers épithéliaux.

\section{Avantages/inconvénients et règlementation des associations d'anticorps}

L'homo- ou l'hétéro-combinaison d'anticorps présente de nombreux avantages par rapport à la monothérapie anticorps (Tableau III). La combinaison d'anticorps permet de cibler plusieurs épitopes bien définis présents sur un ou plusieurs antigènes, avec un rapport stæchiométrique d'anticorps parfaitement contrôlé et ajustable. Au sein d'un cocktail, chaque anticorps peut être adapté en termes d'affinité, d'épitope, d'isotype, ou de glycosylation. Le fait de cibler plusieurs épitopes sur un même récepteur ou un pathogène, ou sur plusieurs récepteurs ou souches de pathogènes, permet également d'augmenter le nombre de cibles recrutées et donc le nombre d'anticorps liés par entité biologique (cellule ou pathogène). Les réponses biologiques induites par ces anticorps s'en trouvent renforcées, comme les mécanismes immunitaires effecteurs dépendant de la région $\mathrm{Fc}$ de l'IgG (ADCC, CDC [complement-dependent cytotoxicity], ADCP [antibodydependent cell phagocytosis]), l'inhibition d'une signalisation cellulaire compensatoire (éventuellement par l'élimination ou l'internalisation/dégradation de la, ou des cibles) qui repose sur la partie Fab de l'IgG, ou la neutralisation et la clairance de virus. Dans le cas de I'hétéro-combinaison d'anticorps anti-points de contrôle immunitaire ou dirigés contre leurs ligands (comme PDL-1 par exemple), la combinaison permet de contrer simultanément les mécanismes de régulation négative redondants exploités par les tumeurs pour échapper au système immunitaire. Un mélange oligoclonal d'anticorps permet ainsi d'élargir le spectre d'activité du biomédicament, anticipant et évitant d'éventuelles résistances au traitement, préexistantes ou acquises par émergence de clones résistants sous l'effet d'un des constituants de l'association. Enfin, en cancérologie, les hétéro-combinaisons ciblant la cellule cancéreuse ou le microenvironnement tumoral immunitaire et les vaisseaux, procèdent de mécanismes d'action potentiellement synergiques in vivo dont les modalités restent à clarifier.

La production des mélanges d'anticorps est globalement régie selon les bonnes pratiques de fabrication classiquement utilisées pour produire les anticorps thérapeutiques [51]. Actuellement, la stratégie de «formulation séparée » continue à prévaloir pour associer deux AcM, que ce soit dans les formulations déjà approuvées ou en cours d'essais cliniques. Toutefois, cette stratégie sera de plus en plus coûteuse si on souhaite développer des mélanges de trois à six anticorps, avec des procédures et des contraintes de réglementation de plus en plus lourdes et compliquées. En effet, les règlementations de l'EMA (agence européenne des médicaments) et de la FDA imposent d'évaluer les composants d'un mélange oligoclonal individuellement et en combinaison, pour leur toxicité, leur efficacité et leur pharmaco-cinétique. Les procédures règlementaires sont donc dédoublées pour chaque anticorps "principe actif», chacun étant considéré comme un biomédicament unique dans une association. Face à ces difficultés, les stratégies «production en cellule unique » ou «production par un mélange de plusieurs cellules » devraient permettre de maîtriser les procédés et coûts de production, et de faciliter les procédures réglementaires et d'enregistrement [52]. Concrètement, la FDA a déjà autorisé des mélanges oligoclonaux d'anticorps, préparés selon la stratégie «formulation unique », à être utilisés dans des tests cliniques de phase I et II [44]. Des essais de phase I avec le Sym004 [53] et le MM-151 [54], obtenus selon la stratégie «formulation unique », ont obtenu l'autorisation de la FDA pour être testés comme «biomédicament unique » en cancérologie. II est donc 


\begin{tabular}{|c|c|c|c|c|c|}
\hline Indication thérapeutique & Anticorps & Formulation & Cibles & $\begin{array}{l}\text { Phase } \\
\text { clinique }\end{array}$ & Date \\
\hline Cancer colorectal/cancer du poumon & $M M-151$ & Unique & $3 \times \varepsilon G F R$ & Phase I* & 2015 \\
\hline Cancer colorectal/cancer du poumon & $M M-151+M M-121$ & Séparée & $3 \times \varepsilon G F R+H \varepsilon R 3$ & Phase I* & 2015 \\
\hline Cancer colorectal/glioblastome & Sym004: futuximab + modotuximab & Unique & $2 \times \varepsilon G F R$ & Phase II & 2018 \\
\hline Cancers épithéliaux & Sym013 & Plusieurs cellules & $\begin{array}{l}2 x \varepsilon G F R+2 x H \varepsilon R 2 \\
+2 x \text { HER3 }\end{array}$ & Phase II* & 2016 \\
\hline Tumeurs cMET-amplifié & Sym015 & Plusieurs cellules & $2 \times \mathrm{xCET}$ & Phase II & 2016 \\
\hline Cancer du sein & Bispécifique MCLA-128 & Cellule unique & HER2/HER3 & Phase I & 2017 \\
\hline Leucémie myéloïde aiguë & Bispécifique MCLA-117 & Cellule unique & $\mathrm{CLECl2A/CD3}$ & Phase I & 2017 \\
\hline Cancer colorectal & Bispécifique MCLA-158 & Cellule unique & LGR5/عGFR & Phase I & 2018 \\
\hline
\end{tabular}

Tableau II. Principaux essais cliniques utilisant un mélange d'anticorps en oncologie. *Développement stoppé.

nécessaire d'évaluer très précocement, au stade préclinique, la stratégie de production de mélanges oligoclonaux pour réduire les risques et les coûts. Reste à savoir comment la FDA ou l'EMA appréhenderont d'un point de vue règlementaire les nouveaux mélanges oligoclonaux d'anticorps préparés selon les stratégies de «production en cellule unique » ou de «production par un mélange de cellules».

Un mélange oligoclonal est formulé selon un rapport stæchiométrique d'anticorps défini lors de la demande règlementaire initiale d'«investigational new drug $»^{3}$. Du fait de la pharmacocinétique différentielle de chaque anticorps (absorption, distribution, métabolisme et excrétion), la formulation stœchiométrique ne sera probablement pas maintenue chez le patient traité. Ce problème doit être appréhendé dès les études précliniques. La sélection de la dose et de la séquence de traitement chez l'homme doit tenir compte de la pharmacocinétique particulière de chaque anticorps dans le mélange. Ainsi, le rapport stœechiométrique des six anticorps constituant le mélange Sym013 variera in vivo au cours du temps en fonction de l'exposition à la cible, forte pour l'EGFR, ou allant de moyenne à faible pour HER2/Neu et HER3. Cela sera encore plus vrai pour des mélanges oligoclonaux antiinfectieux pour lesquels l'élimination in vivo du pathogène dépend de multiples facteurs. La potentialisation de la toxicité, observée dans certains essais cliniques de combinaison [10], souligne la nécessité de bien ajuster le choix de la dose dans les essais cliniques de phase I. Celle-ci ne sera pas obligatoirement calquée sur la dose approuvée en monothérapie. Actuellement, les deux combinaisons approuvées en clinique utilisent les doses d'anticorps identifiés dans les essais cliniques en monothérapie. La caractérisation des mécanismes d'action propres au mélange oligoclonal, en comparaison avec chaque AcM présent dans le mélange, devra être réalisée précocément dans des

\footnotetext{
${ }^{3}$ Une autorisation réglementaire est requise pour la réalisation d'essais cliniques. Aux États-Unis, une
} demande d'investigational new drug (ou IND) doit être déposée auprès de la FDA. modèles précliniques pertinents afin de bien étayer le développement clinique.

\section{Conclusion : vers un mime de la réponse immunitaire polyclonale thérapeutique?}

Le système immunitaire a naturellement évolué pour développer une réponse humorale polyclonale. Le développement d'associations ou de mélanges oligoclonaux d'anticorps pour améliorer les thérapies ciblées existantes aborde un tournant décisif. II devrait permettre de mimer cette réponse immunitaire humorale. Toutefois, des contraintes biologiques, pratiques et règlementaires, doivent être franchies pour enrichir la classe pharmacologique des anticorps avec ce nouveau concept. $\diamond$

\section{SUMMARY}

Mimicking polyclonal immune response in therapy: from combination of two monoclonal antibodies to oligoclonal antibody-based mixtures

Monoclonal antibodies have revolutionized the treatment of many diseases, but their clinical effectiveness remains limited in some cases. Associations of antibodies binding to the same target (homo-combination) or to several different targets (hetero-combination), thereby mimicking a polyclonal humoral immune response, have demonstrated a therapeutic improvement in preclinical and clinical trials, mainly in the field of oncology and infectious diseases. The combinations increase the efficacy of the biological responses and override resistance mechanisms observed with antibody monothe- 


\begin{tabular}{lll}
\hline & Avantages & Inconvénients \\
& $\begin{array}{l}\text { Ciblage de plusieurs épitopes } \\
\text { Synergie fonctionnelle: }\end{array}$ & Production des mélanges : \\
& aggrégation & réglementation \\
Combinaison & activation/inhibition du système immunitaire & Toxicité \\
d'anticorps & activation/inhibition de la signalisation Fab-dépendante & Pharmacocinétique différentielle de chaque anticorps \\
& Élimination de la cible & La formulation stœchiométrique \\
& Diminution de la résistance aux traitements & La dose et la fréquence d'injection
\end{tabular}

Tableau III. Avantages et inconvénients des mélanges d'anticorps.

rapy. The most common method of formulating and administering antibody combinations is a separate formulation, with sequential injection of each antibody as individual drug substance. Alternatively, combined formulations are developed where the separately-produced antibodies are mixed before administration or produced simultaneously by a single cell line, or a mixture of cell lines as a polyclonal master cell bank. The regulation, the toxicity and the injection sequence of these oligoclonal antibody-based mixtures remain points to be clarified and optimized for a better therapeutic effect. $\diamond$

\section{REMERCIEMENTS}

Les auteurs remercient André Pèlegrin, Isabelle Navarro-Teulon, Bruno Robert, Nadège Gaborit, Yassamine Lazrek et Gaëlle Thomas pour leur contribution aux travaux de l'équipe auxquels il est fait référence dans cet article; ils remercient également Véronique Garambois, Geneviève Heintz, Sabine Bousquié, et toute l'équipe de l'animalerie de l'IRCM pour leur assistance technique. Ces travaux ont été financés par la Ligue contre le Cancer, le cancéropôle GSO, le GEFLUC, le programme FUII3 UmAbHER3 (BPI France), le SIRIC Montpellier Cancer, Inserm Transfert et le LabEx MAbImprove ANR-10-LABX-53-01.

\section{LIENS D'INTÉRÊT}

Christel Larbouret, Marie-Alix Poul et Thierry Chardès déclarent avoir participé à des interventions ponctuelles (collaboration pour des travaux scientifiques) avec les sociétés Roche, LFB, CisBio et GamaMabs.

\section{RÉFÉRENCES}

1. Friedman LM, Rinon A, Schechter B, et al. Synergistic down-regulation of receptor tyrosine kinases by combinations of mAbs: implications for cancer immunotherapy. Proc Natl Acad Sci USA $2005 ; 102: 1915-20$.

2. Ben-Kasus T, Schechter B, Lavi S, et al. Persistent elimination of ErbB-2/HER2-overexpressing tumors using combinations of monoclonal antibodies: relevance of receptor endocytosis. Proc Natl Acad Sci USA 2009 ; 106 : 3294-9.

3. Poulsen TT, Grandal MM, Skartved NJØ, et al. Sym015: a highly efficacious antibody mixture against MET-amplified tumors. Clin Cancer Res 2017 ; 23 : 5923-35.

4. lida M, Brand TM, Starr MM, et al. Overcoming acquired resistance to cetuximab by dual targeting HER family receptors with antibody-based therapy. Mol Cancer $2014 ; 13: 242$.

5. Pollmann SE, Calvert VS, Rao S, et al. Acquired resistance to a MET antibody in vivo can be overcome by the MET antibody mixture Sym015. Mol Cancer Ther 2018; $17: 1259-70$.

6. Arena S, Siravegna G, Mussolin B, et al. MM-151 overcomes acquired resistance to cetuximab and panitumumab in colorectal cancers harboring EGFR extracellular domain mutations. Sci Transl Med 2016 ; 8 : 324ral4.

7. Larbouret C, Robert B, Navarro-Teulon I, et al. In vivo therapeutic synergism of anti-epidermal growth factor receptor and anti-HER2 monoclonal antibodies against pancreatic carcinomas. Clin Cancer Res 2007 ; $13: 3356-62$.
8. Thomas G, Chardès T, Gaborit N, et al. HER3 as biomarker and therapeutic target in pancreatic cancer: new insights in pertuzumab therapy in preclinical models. Oncotarget $2014 ; 5: 7138-48$.

9. Maron R, Schechter B, Mancini M, et al. Inhibition of pancreatic carcinoma by homo- and heterocombinations of antibodies against $\varepsilon \mathrm{GF}$-receptor and its kin HER2/ErbB-2. Proc Natl Acad Sci USA 2013; 110 : 15389-94.

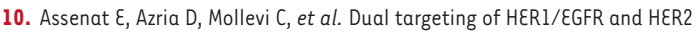
with cetuximab and trastuzumab in patients with metastatic pancreatic cancer after gemcitabine failure: results of the THERAPY phase 1-2 trial. Oncotarget $2015 ; 6: 12796-808$.

11. Van Cutsem $\varepsilon$, Eng C, Nowara $\varepsilon$, et al. Randomized phase lb/II trial of rilotumumab or ganitumab with panitumumab versus panitumumab alone in patients with wild-type KRAS metastatic colorectal cancer. Clin Cancer Res $2014 ; 20: 4240-50$.

12. Mancini M, Gal H, Gaborit N, et al. An oligoclonal antibody durably overcomes resistance of lung cancer to third-generation EGFR inhibitors. EMBO Mol Med 2018; $10: 294-308$.

13. Jacobsen $H J$, Poulsen $T$, Dahlman $A$, et al. Pan-HER, an antibody mixture simultaneously targeting $\varepsilon G F R, H \varepsilon R 2$, and HER3, effectively overcomes tumor heterogeneity and plasticity. Clin. Cancer Re $2015 ; 21: 4110-22$.

14. Strauss SJ, Morschhauser F, Rech J, et al. Multicenter phase II trial of immunotherapy with the humanized anti-CD22 antibody, epratuzumab, in combination with rituximab, in refractory or recurrent non-Hodgkin's lymphoma. J Clin Oncol 2006 ; 24 : 3880-6.

15. Volk WA, Synder RM, Benjamin DC, et al. Monoclonal antibodies to the glycoprotein of vesicular stomatitis virus: comparative neutralizing activity. J Virol $1982 ; 42: 220-7$.

16. Bar-On Y, Gruell H, Schoofs T, et al. Safety and antiviral activity of combination HIV-1 broadly neutralizing antibodies in viremic individuals. Nat Med 2018 ; 24 : 1701-7.

17. Qiu X, Audet J, Lv M, et al. Two-mAb cocktail protects macaques against the Makona variant of Ebola virus. Sci Transl Med 2016 ; 8 : 329ra33.

18. Galun $\varepsilon$, Eren R, Safadi R, et al. Clinical evaluation (phase I) of a combination of two human monoclonal antibodies to HBV: safety and antiviral properties. Hepatology 2002 ; 35 : 673-9.

19. Lantto J, Haahr Hansen M, Rasmussen SK, et al. Capturing the natural diversity of the human antibody response against vaccinia virus. J Virol $2011 ; 85: 1820-33$.

20. Pascal KE, Coleman CM, Mujica AO, et al. Pre- and postexposure efficacy of fully human antibodies against Spike protein in a novel humanized mouse model of MERS-CoV infection. Proc Natl Acad Sci USA 2015 ; 112 : 8738-8743.

21. Bakker ABH, Marissen WE, Kramer RA, et al. Novel human monoclonal antibody combination effectively neutralizing natural rabies virus variants and individual in vitro escape mutants. J Virol $2005 ; 79$ : 9062-8.

22. Marozsan AJ, Ma D, Nagashima KA, et al. Protection against Clostridium difficile infection with broadly neutralizing antitoxin monoclonal antibodies. J Infect Dis $2012 ; 206$ : 706-13.

23. Fan Y, Garcia-Rodriguez C, Lou J, et al. A three monoclonal antibody combination potently neutralizes multiple botulinum neurotoxin serotype $F$ subtypes. PLoS One2017 ; 12 : e0174187. 


\section{RÉFÉRENCES}

24. Nguyen AW, Wagner EK, Laber JR, et al. A cocktail of humanized anti-pertussis toxin antibodies limits disease in murine and baboon models of whooping cough. Sci Transl Med $2015 ; 7$ : 316 ral95.

25. Curran MA, Montalvo W, Yagita H, et al. PD-1 and CTLA-4 combination blockade expands infiltrating T cells and reduces regulatory T and myeloid cells within B16 melanoma tumors. Proc Natl Acad Sci USA $2010 ; 107:$ 4275-80.

26. Chen S, Lee L-F, Fisher TS, et al. Combination of 4-1BB agonist and PD-1 antagonist promotes antitumor effector/memory CD8 T cells in a poorly immunogenic tumor model. Cancer Immunol Res $2015 ; 3: 149-60$.

27. Guo Z, Cheng D, Xia Z, et al. Combined TIM-3 blockade and CD137 activation affords the longterm protection in a murine model of ovarian cancer. J Transl Med 2013; $11: 215$.

28. Chaganty BKR, Qiu S, Gest A, et al. Trastuzumab upregulates PD-LI as a potential mechanism of trastuzumab resistance through engagement of immune effector cells and stimulation of IFN $\gamma$ secretion. Cancer Lett $2018 ; 430: 47-56$.

29. Baselga J, Cortés J, Kim SB, et al. Pertuzumab plus trastuzumab plus docetaxel for metastatic breast cancer. $N$ Engl J Med $2012 ; 366: 109-19$

30. Minckwitz $\mathrm{G}$ von, Procter M, Azambuja $\varepsilon$ de, et al. Adjuvant pertuzumab and trastuzumab in early HER2-positive breast cancer. $N$ Engl J Med 2017 ; 377 : 122-31.

31. Tabernero J, Hoff PM, Shen L, et al. Pertuzumab plus trastuzumab and chemotherapy for HER2positive metastatic gastric or gastro-oesophageal junction cancer (JACOB): final analysis of a double-blind, randomised, placebo-controlled phase 3 study. Lancet Oncol 2018 ; 19 : 1372-84.

32. Tol J, Koopman M, Cats A, et al. Chemotherapy, bevacizumab, and cetuximab in metastatic colorectal cancer. N Engl J Med 2009; $360: 563-72$

33. Hecht JR, Mitchell $\varepsilon$, Chidiac T, et al. A randomized phase IIIB trial of chemotherapy, bevacizumab, and panitumumab compared with chemotherapy and bevacizumab alone for metastatic colorectal cancer. J Clin Oncol 2009 ; 27 : 672-80.

34. Gianni L, Romieu GH, Lichinitser M, et al. AVEREL: a randomized phase III Trial evaluating bevacizumab in combination with docetaxel and trastuzumab as first-line therapy for HER2 positive locally recurrent/metastatic breast cancer.J Clin Oncol 2013; $31: 1719-25$.

35. Heskamp S, Boerman OC, Molkenboer-Kuenen JDM, et al. Cetuximab reduces the accumulation of radiolabeled bevacizumab in cancer xenografts without decreasing VEGF expression. Mol Pharm $2014 ; 11: 4249-57$.

36. Wolchok JD, Kluger H, Callahan MK, et al. Nivolumab plus ipilimumab in advanced melanoma. N EnglJ Med $2013 ; 369: 122-33$

37. Wolchok JD, Chiarion-Sileni V, Gonzalez R, et al. Overall survival with combined nivolumab and ipilimumab in advanced melanoma. N Engl J Med 2017 ; 377 : 1345-56.

38. Larkin J, Chiarion-Sileni V, Gonzalez R, et al. Combined nivolumab and ipilimumab or monotherapy in untreated melanoma. N Engl J Med $2015 ; 373: 23-34$.

39. Socinski MA, Jotte RM, Cappuzzo F, et al. Atezolizumab for first-line treatment of metastatic nonsquamous NSCLC. N Engl J Med 2018 ; $378: 2288-301$.

40. Henricks LM, Schellens JHM, Huitema ADR, et al. The use of combinations of monoclonal antibodies in clinical oncology. Cancer Treat. Rev 2015; $41: 859-67$.

41. Corti D, Kearns JD. Promises and pitfalls for recombinant oligoclonal antibodies-based therapeutics in cancer and infectious disease. Curr Opin Immunol 2016; 40:51-61.
42. Chae YK, Arya A, lams W, et al. Current landscape and future of dual antiCTLA4 and PD-1/PD-L1 blockade immunotherapy in cancer; lessons learned from clinical trials with melanoma and non-small cell lung cancer (NSCLC).J Immunother Cancer 2018; $6 \mathrm{~h} 39$.

43. Pedersen MW, Jacobsen HJ, Koefoed K, et al. Sym004: a novel synergistic anti-epidermal growth factor receptor antibody mixture with superior anticancer efficacy. Cancer Res $2010 ; 70: 588-97$.

44. Nayak SU, Griffiss JM, McKenzie R, et al. Safety and pharmacokinetics of XOMA $3 A B$, a novel mixture of three monoclonal antibodies against botulinum toxin A. Antimicrob. Agents Chemother $2014 ; 58: 5047-53$.

45. Napolitano S, Martini G, Martinelli $\varepsilon$, et al. Antitumor efficacy of triple monoclonal antibody inhibition of epidermal growth factor receptor (EGFR) with MM151 in EGFR-dependent and in cetuximab-resistant human colorectal cancer cells. Oncotarget $2017 ; 8: 82773-83$.

46. Kruif J de, Kramer A, Nijhuis R, et al. Generation of stable cell clones expressing mixtures of human antibodies. Biotechnol Bioeng 2010 ; $106: 741-50$.

47. Geuijen CAW, De Nardis C, Maussang D, et al. Unbiased combinatorial screening identifies a bispecific $\operatorname{lgGl}$ that potently inhibits HER3 signaling via HER2-guided ligand blockade. Cancer Cell 2018; 33 : 922-936.el0.

48. Rasmussen SK, Nielsen LS, Müller C, et al. Recombinant antibody mixtures; optimization of cell line generation and single-batch manufacturing processes. BMC Proc 2011; 5 (suppl 8) : 02.

49. Wiberg FC, Rasmussen SK, Frandsen TP, et al. Production of target-specific recombinant human polyclonal antibodies in mammalian cells. Biotechnol Bioeng 2006 ; $94: 396-405$.

50. Robak T, Windyga J, Trelinski J, et al. Rozrolimupab, a mixture of 25 recombinant human monoclonal $\mathrm{RhD}$ antibodies, in the treatment of primary immune thrombocytopenia. Blood $2012 ; 120: 3670-6$.

51. Chon JH, Zarbis-Papastoitsis G. Advances in the production and downstream processing of antibodies. N Biotechnol $2011 ; 28: 458-63$.

52. Rasmussen SK, Næsted H, Müller C, et al. Recombinant antibody mixtures: production strategies and cost considerations. Arch Biochem Biophys 2012 ; $526: 139-45$

53. Kojima T, Yamazaki K, Kato K, et al. Phase I dose-escalation trial of Sym004, an anti-EGFR antibody mixture, in Japanese patients with advanced solid tumors. Cancer Sci. $2018 ; 109$ : 3253-62.

54. Lieu CH, Harb WA, Beeram M, et al. Phase 1 trial of MM-151, a novel oligoclonal anti-EGFR antibody combination in patients with refractory solid tumors. JCO $2014 ; 32: 2518$.

55. Carvalho S, Levi-Schaffer F, Sela M, et al. Immunotherapy of cancer: from monoclonal to oligoclonal cocktails of anti-cancer antibodies: IUPHAR Review 18. BrJ Pharmacol 2016 ; 173 : 1407-24.

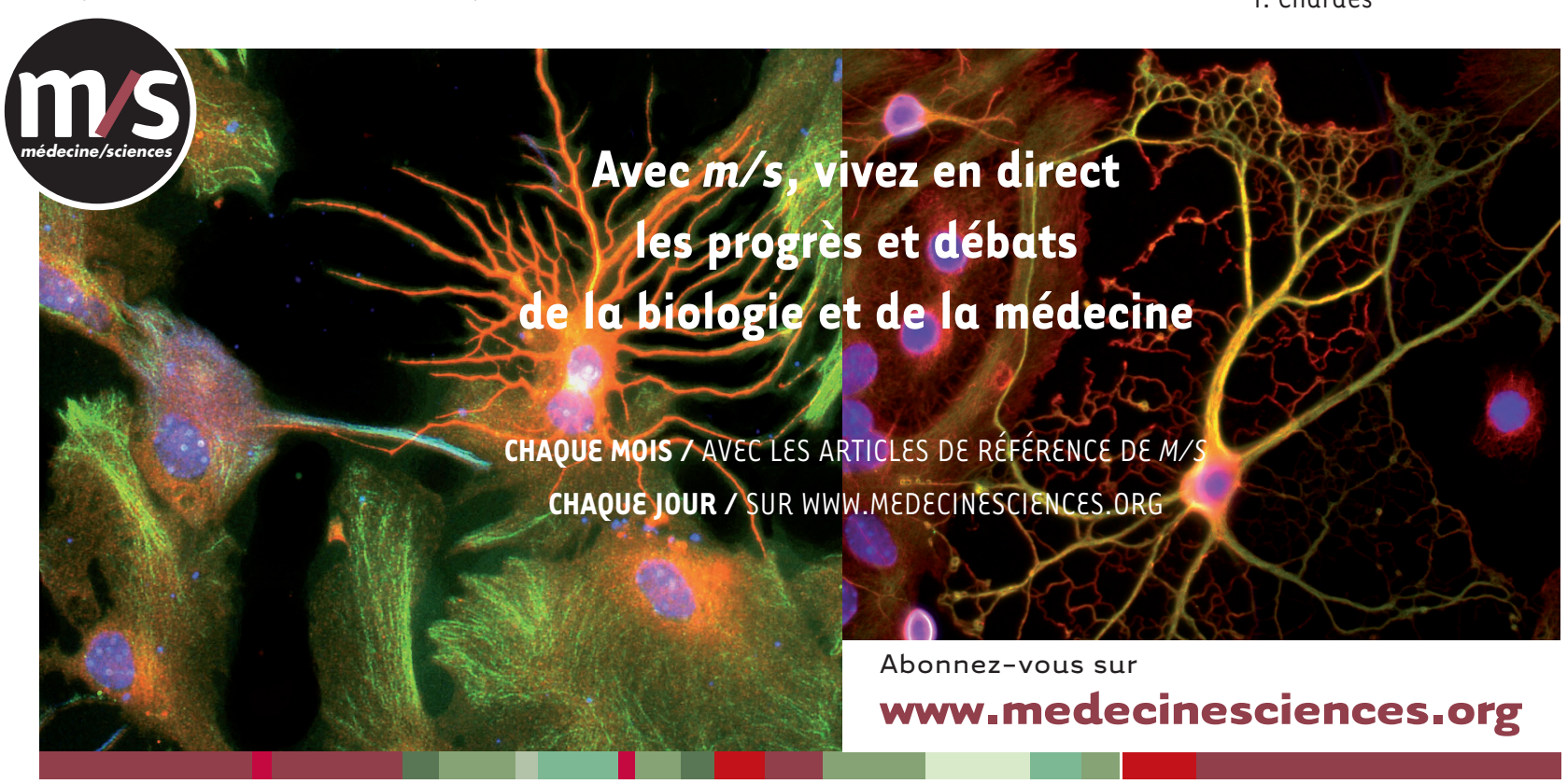

